\title{
General Psychiatry Relationships between childhood trauma and dissociative, psychotic symptoms in patients with schizophrenia: a case-control study
}

\author{
Tugce Taskin Uyan, ${ }^{1}$ Mehmet Baltacioglu, ${ }^{1}$ Cicek Hocaoglu ${ }^{2}$
}

To cite: Uyan TT, Baltacioglu M, Hocaoglu C. Relationships between childhood trauma and dissociative, psychotic symptoms in patients with schizophrenia: a case-control study. General Psychiatry 2022;35:e100659. doi:10.1136/ gpsych-2021-100659

Received 11 September 2021 Accepted 29 December 2021

Check for updates

(C) Author(s) (or their employer(s)) 2022. Re-use permitted under CC BY-NC. No commercial re-use. See rights and permissions. Published by BMJ.

${ }^{1}$ Clinic for Psychiatry, Rize State Hospital, Rize, Turkey

${ }^{2}$ Department of Psychiatry, Faculty of Medicine, Recep Tayyip Erdoğan University, Rize, Turkey

Correspondence to Professor Cicek Hocaoglu; cicekh@gmail.com

\section{ABSTRACT}

Background Childhood trauma (CT) is an important risk factor in the emergence and clinical course of psychiatric disorders. In the latest literature, an association exists between CT and patients with schizophrenia. There is also a strong relationship between the dissociative symptoms of schizophrenia and the presence of CT.

Aims The aim of this study is to examine the relationship between CT and dissociative, positive and negative symptoms in patients with schizophrenia.

Methods One hundred patients with schizophrenia and 100 healthy volunteers were included in the study. The Sociodemographic Data Form, Dissociative Experiences Scale (DES), Positive and Negative Syndrome Scale (PANSS), and Childhood Trauma Questionnaire (CTQ) were administered to all participants.

Results The CTQ and DES scores of the schizophrenia group were significantly higher than those of the control group. In patients with schizophrenia, a positive association was found between positive symptoms and DES scores. In terms of negative symptoms, a positive association was found between apathetic social withdrawal and CTQ-emotional neglect (EN), CTQ-physical neglect (PN) and CTQ total scores. There was a significant positive correlation between CTQ-EN scores and negative symptoms and PANSS scores. No significant relationship was found between negative symptoms and DES scores. Conclusions High rates of CT and dissociative symptoms are seen in patients with schizophrenia. In addition, the findings of the relationship between CT and dissociative, positive and negative symptoms are also noteworthy. Therefore, it may be important for clinicians to assess trauma history during the psychiatric evaluation of patients with schizophrenia.

\section{INTRODUCTION}

Schizophrenia is a neurodevelopmental process that begins in late adolescence and early adulthood, affecting approximately $1 \%$ of the population. A significant decrease in quality of life and functionality is observed in individuals diagnosed with schizophrenia. It is characterised by positive, negative, affective and cognitive symptoms. ${ }^{1}$

Both dissociative experiences and CT are quite common in schizophrenia. ${ }^{2}$ The causal relationship between CT and schizophrenia has been investigated for many years. Recent studies have also focused on this area. ${ }^{34}$ In these studies, CT was found to increase the risk of psychosis development in adulthood and even negatively affect the course of the disease (such as earlier, longer, or higher rates of hospitalisation and increased suicide risk). ${ }^{56}$ One study which investigated the risk of psychosis development with exposure to trauma in childhood stated that CT increased the risk of psychosis development approximately 1.7-15 times. ${ }^{7}$ Considering the studies, it has been reported that the situation is similar in patients diagnosed with schizophrenia. It has been reported that CT is observed at a rate of $45 \%$ to $85 \%$ in patients with schizophrenia compared to the control group. ${ }^{3-8}$

The dissociative experiences of schizophrenia can be seen as a defence mechanism against mental traumas such as emotional, physical and sexual neglect or abuse, especially for those occurring in childhood. Childhood trauma (CT) occurs suddenly and unexpectedly, threatening the physical or mental integrity of the person; it is defined as a psychologically challenging and traumatic experience that overturns one's coping skills and defence mechanisms. ${ }^{7}$ CT can result from sexual, emotional and physical abuse or physical and emotional neglect, as well as the loss of parents at an early age, separation, migration, witnessing death or violence, being involved in an accident, or being exposed to natural disasters. ${ }^{8}$

Read et al reported a strong relationship between hallucinations and delusions and CT in their literature review in which they compiled the results of 51 studies that addressed the relationship between childhood traumatic experiences and psychotic disorders. ${ }^{9}$ One study conducted with 569 patients with schizophrenia found that the frequency of childhood traumatic life experiences was higher than within the normal population. ${ }^{10}$ 
A systematic review of 46 studies investigating the relationship between psychotic disorders and CT found the prevalence of CT in patients with psychosis ranged from $28 \%$ to $73 \%$, sexual abuse rates ranged from $13 \%$ to $61 \%$, and physical abuse rates ranged from $10 \%$ to $61 \% .{ }^{11}$ In a study in which patients diagnosed with first-episode psychosis were evaluated, it was reported that $52 \%$ of the patients had at least one experience of childhood abuse and $43 \%$ had at least one experience of childhood neglect. ${ }^{6}$

A study by Vogel et al reported that a strong relationship exists between CT and dissociative symptoms in patients with schizophrenia. ${ }^{12}$ Ross and Keyes defined a type of schizophrenia, which they called 'dissociative schizophrenia' or 'dissociative subtype of schizophrenia' based on CT history, claiming a direct relationship between dissociation and schizophrenia. ${ }^{13}$

Looking at these findings, the high rate of CT history in patients with schizophrenia and other psychotic-related illnesses suggests that CT may be influential in the aetiology of schizophrenia.

The aim of this study was to contribute to the current literature by examining the relationship between CT and dissociative, positive and negative symptoms in patients with schizophrenia.

\section{METHODS}

\section{Subjects}

Subjects were recruited from the Training and Research Hospital Psychiatry Outpatient Clinic and the State Hospital Community Mental Health Center consecutively between 1 July 2016 and 1 December 2017. A total of 107 patients who met the inclusion criteria - a diagnosis of schizophrenia according to the Diagnostic and Statistical Manual of Mental Disorders, Fifth Edition criteria and aged between 18 and 64 years - were included in the study. Patients with dementia, neurological disorders, alcohol-substance use disorders, or mental retardation, and pregnant or breastfeeding women were excluded from the evaluation. Seven of the patients with schizophrenia originally enrolled in the study were excluded: four of the subjects did not complete the questionnaires, and three subjects did not wish to participate in the study. The study was completed with a total of 100 patients.

The control group consisted of 100 healthy participants, including hospital staff, who had similar characteristics as the study group; they did not have any neurological or psychiatric illnesses and voluntarily agreed to participate in the study. Overall, 100 patients (51 women and 49 men) and 100 healthy controls (65 women and 35 men) completed the study. In the schizophrenia group, the mean age of onset of the disease was 23.45 (7.43) years and the mean disease duration was 18.41 (8.85) years. The flowchart is shown in figure 1 .

\section{Study tools}

\section{Sociodemographic Data Form}

The Sociodemographic Data Form, prepared by the researchers of this study, was used to gather information on age, gender, birthplace, marital status, educational status, occupation, socioeconomic status, age of illness onset, duration of illness, number of hospitalisations, and the psychiatric history of first-degree relatives. The information collected provided the independent variables for the research, including the drugs used by patients.

\section{Childhood Trauma Questionnaire (CTQ)}

CTQ is a self-report scale consisting of 28 questions developed by Bernstein et al in 1995 that retrospectively scans the abuse and neglect experiences before the age of 20 years. ${ }^{14}$ It has five subdimensions: emotional, physical and

Figure 1 Flowchart of the study.

107 outpatients with schizophrenia were treated at the Training and Research Hospital Psychiatry Outpatient Clinic and State Hospital Community Mental Health Center and matched by gender, age, education and location of residence with patients

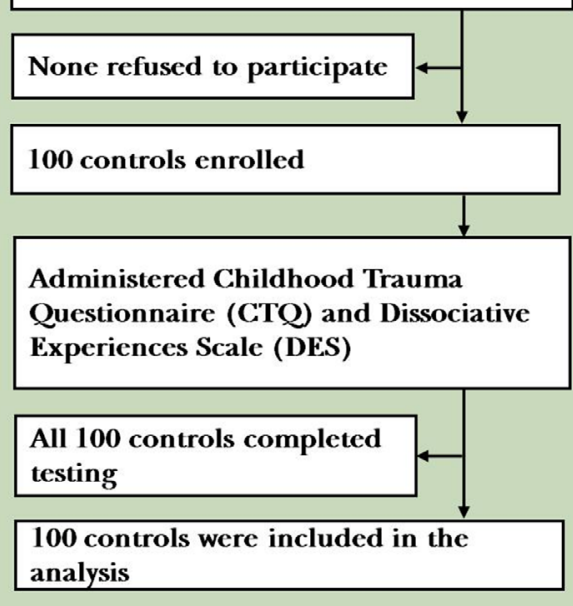

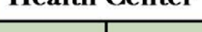

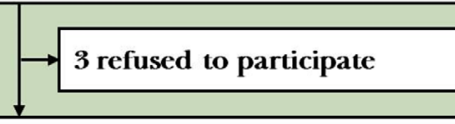

104 patients enrolled

Administered CTQ, DES, the Scale for the Assessment of Negative Symptoms (SANS) and the Scale for the

Assessment of Positive Symptoms (SAPS) 4 did not complete testing

100 patients were included in the
analysis


sexual abuse, and emotional and physical neglect. The validity and reliability study of the Turkish version of the scale was conducted by Sar et al in 2012. ${ }^{15}$

\section{Dissociative Experiences Scale (DES)}

DES is a 28-item scale, developed by Bernstein and Putnam, used to screen dissociative experiences and disorders and to measure their severity. ${ }^{16}$ A subject's score ranges from 0 to 100 for each item of the scale, and the result is obtained by calculating the average of the total scores. Scores of 30 and above indicate the presence of a dissociative disorder. The validity and reliability study of the Turkish version of the scale was conducted by Sar et al in $1997 .^{17}$

\section{Positive and Negative Syndrome Scale (PANSS)}

PANSS was developed by Kay et al in $1987 .{ }^{18}$ The 30 -item scale provides information about the presence and severity of positive and negative symptoms in patients with schizophrenia, as well as their general level of psychopathology. The validity and reliability study of this scale was conducted by Kostakoglu et al in Turkey in $1999 .{ }^{19}$

\section{Statistical analysis}

SPSS Statistics for Windows, V.23.0 (IBM SPSS Statistics for Windows) was used to evaluate the data in this study. Descriptive analyses were given using mean (SD) for continuous variables and $\mathrm{n}(\%)$ for categorical variables. The conformity of the variables to the normal distribution was evaluated with the Kolmogorov-Smirnov test. For comparisons of categorical variables, the Pearson $\chi^{2}$ test was used. The relationships between continuous variables were evaluated with the Pearson correlation analysis. Logistic regression analysis was performed on the gender variable and the scores of emotional abuse (EA), emotional neglect (EN), physical neglect (PN), physical abuse (PA), sexual abuse (SA), and CTQ scales applied to the schizophrenia and control groups. In this way, an explanatory model was tried to be created by determining the variables that are important in determining whether the patients are at risk of schizophrenia or not. In the above-mentioned binary logistic regression analysis, with the patient and control groups as the dependent variable, the model was created by using the CTQ and its subscales scores and Dissociative Experiences Scale (DES)scores as independent variables. Whether there is any mediator variable from the effect of CT on the development of schizophrenia was evaluated by mediation model regression analysis. A $p$ value of $<0.05$ was considered statistically significant.

\section{RESULTS}

Demographic and clinical characteristics of the study groups Table 1 presents the demographic and clinical characteristics of the study groups. One hundred patients with schizophrenia and 100 healthy controls were included in the study. Accordingly, no significant difference was found between the two groups in terms of age and gender. However, there was a significant difference between the groups in terms of the marital status $\left(\chi^{2}=12.602, \mathrm{p}<0.001\right)$ and employment status $\left(\chi^{2}=109.695, \mathrm{p}<0.001\right)$ distribution. Also, the frequency of smoking in the schizophrenia group was significantly higher than in the control group $\left(\chi^{2}=14.581, p<0.001\right)$. Additionally, in the patients with schizophrenia, the frequency of receiving continuous treatment $\left(\chi^{2}=109.397, \mathrm{p}<0.001\right)$ and the presence of psychiatric disorders in relatives $\left(\chi^{2}=59.318\right.$, $\mathrm{p}<0.001)$ were significantly higher, while the level of education was lower $\left(\chi^{2}=74.261, \mathrm{p}<0.001\right)$ when compared to control group members.

\section{Scale scores and patient/control group comparisons}

Table 2 compares the DES and CTQ total and subscale scores of patients with schizophrenia and healthy controls.

Table 1 Demographic and clinical characteristics of the study groups

\begin{tabular}{|c|c|c|c|c|}
\hline Characteristics & $\begin{array}{l}\text { Patients with } \\
\text { schizophrenia } \\
(n=100)\end{array}$ & $\begin{array}{l}\text { Healthy } \\
\text { controls } \\
(n=100)\end{array}$ & Statistics & $P$ value \\
\hline Age (years), mean (SD) & $42.38(15.38)$ & $40.60(14.9)$ & $t=-0.703 \dagger$ & 0.482 \\
\hline Marital status (married/single) & $33 / 67$ & $58 / 42$ & $\chi^{2}=12.602^{*}$ & $<0.001$ \\
\hline Family history of psychiatric disorders $(n=77)$ & $65(84.4 \%)$ & $12(15.6 \%)$ & $\chi^{2}=59.318^{*}$ & $<0.001$ \\
\hline Comorbidities $(n=53)$ & $32(60.4 \%)$ & $21(39.6 \%)$ & $\chi^{2}=3.106^{*}$ & 0.109 \\
\hline Regular psychiatric treatment of any kind $(n=72)$ & $72(100.0 \%)$ & $0(0.0 \%)$ & $\chi^{2}=109.397 \ddagger$ & $<0.001$ \\
\hline Smoking $(n=99)$ & $63(63.6 \%)$ & $36(36.4 \%)$ & $\chi^{2}=14.581^{*}$ & $<0.001$ \\
\hline
\end{tabular}

${ }^{*} \chi^{2}$ value.

†t value.

‡Fisher's exact test value. 
Table 2 Comparison of the study groups with DES, CTQ and subscales

\begin{tabular}{|c|c|c|c|c|}
\hline Scales & $\begin{array}{l}\text { Patients with schizophrenia } \\
(n=100) \text {, mean (SD) }\end{array}$ & $\begin{array}{l}\text { Healthy controls } \\
(n=100) \text {, mean (SD) }\end{array}$ & U value & $P$ value \\
\hline DES & 21.47 (13.30) & $9.79(7.66)$ & $2178.5^{\star}$ & $<0.001$ \\
\hline CTQ-SA & $5.91(2.38)$ & $5.08(0.36)$ & $4264.5^{\star}$ & $<0.001$ \\
\hline CTQ-EA & $8.18(2.93)$ & $6.26(1.67)$ & $2575.0^{*}$ & $<0.001$ \\
\hline CTQ-EN & $14.73(3.59)$ & $10.91(4.29)$ & $2133.0^{\star}$ & $<0.001$ \\
\hline CTQ-PA & $6.65(2.61)$ & $5.23(0.77)$ & $3048.0^{*}$ & $<0.001$ \\
\hline CTQ- PN & $8.48(2.36)$ & $7.24(2.38)$ & $3346.0^{\star}$ & $<0.001$ \\
\hline CTQ total & $53.92(12.40)$ & 42.79 (8.91) & $2185.0^{\star}$ & $<0.001$ \\
\hline
\end{tabular}

*Mann-Whitney U test.

CTQ, Childhood Trauma Questionnaire; CTQ-EA, CTQ-emotional abuse; CTQ-EN, CTQ-emotional neglect; CTQ-PA, CTQ-physical abuse; CTQ- PN, CTQ-physical neglect; CTQ-SA, CTQ-sexual abuse; DES, Dissociative Experiences Scale.

DES scores of the schizophrenia group were significantly higher than those of the control group ( $\mathrm{U}=2178.5$, $\mathrm{p}<0.001)$. Total and subscale scores of CTQ were also significantly higher in the schizophrenia group than in the control group (all p-values $<0.001$ ). All variables were not normally distributed.
Comparison of the scales and subscales' scores in patients with schizophrenia

In table 3, the correlation of DES, CTQ subscales, Scale for the Assessment of Positive Symptoms (SAPS), Scale for the Assessment of Negative Symptoms (SANS), and some psychotic symptoms of the patients with schizophrenia was

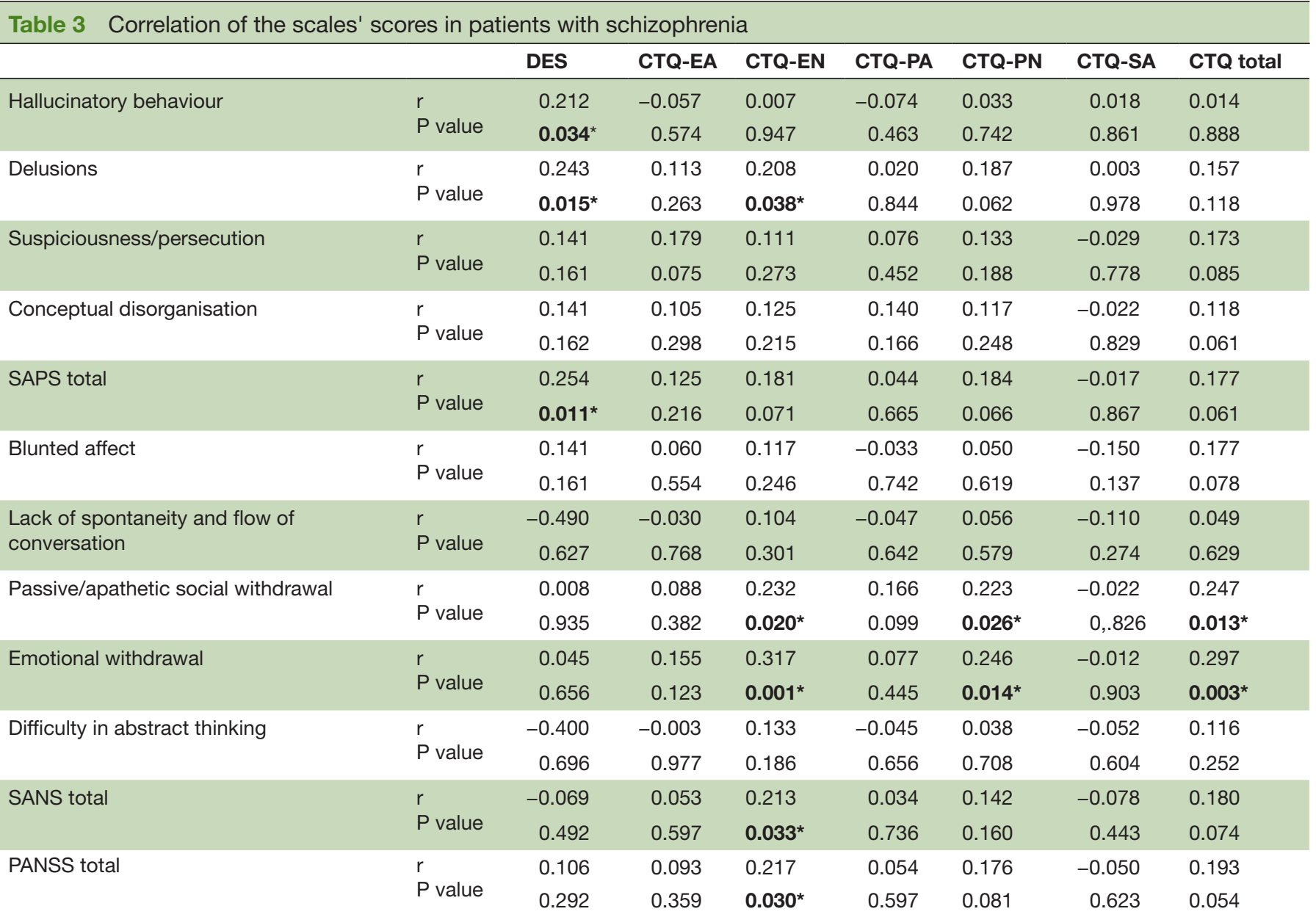

*Pearson correlation test, $\mathrm{p}<0.05$.

CTQ, Childhood Trauma Questionnaire; CTQ-EA, CTQ-emotional abuse; CTQ-EN, CTQ-emotional neglect; CTQ-PA, CTQ-physical abuse; CTQ-PN, CTQ-physical neglect; CTQ-SA, CTQ-sexual abuse; DES, Dissociative Experiences Scale; PANSS, Positive and Negative Syndrome Scale; SANS, Scale for the Assessment of Negative Symptoms; SAPS, Scale for the Assessment of Positive Symptoms. 
Table 4 Multivariate logistic regression model (the backward elimination method based on the likelihood ratio test statistics)

\begin{tabular}{llcll}
\hline & B & Wald & P value & OR (95\% Cl) \\
\hline Gender & 0.992 & 6.909 & 0.009 & 2.696 (1.287 to 5.649$)$ \\
DES & 0.091 & 19.965 & $\mathbf{0 . 0 0 0 1}$ & $1.096(1.052$ to 1.141$)$ \\
CTQ-EN & 0.192 & 13.634 & $\mathbf{0 . 0 0 0 1}$ & $1.212(1.094$ to 1.342$)$ \\
CTQ-PA & 0.548 & 8.923 & $\mathbf{0 . 0 0 3}$ & $1.729(1.207$ to 2.477$)$ \\
CTQ -PN & -0.159 & 2.928 & 0.087 & 0.853 (0.711 to 1.023)
\end{tabular}

log-likelihood value : 182880

CTQ, Childhood Trauma Questionnaire; CTQ-EN, CTQ-emotional neglect; CTQ-PA, CTQ-physical abuse; CTQ-PN, CTQ-physical neglect; DES, Dissociative Experiences Scale.

examined. Two-tailed Pearson correlations were used to explore the associations among CTQ total score, DES, and PANSS scores in the schizophrenia group; significant relationships were demonstrated in all pairs. Further correlation evaluations to ascertain relationships among various forms of CT, schizophrenia and dissociative symptoms showed that greater dissociative symptom severity was significantly associated with higher CTQ total scores, CTQ-PN, CTQ-PA, CTQ-EN, CTQ-EA, CTQ-SA, and more severe hallucinatory behaviour and delusions. Passive/apathetic social withdrawal was positively associated with CTQ-EN $(r=0.232, \mathrm{p}=0.020)$ and CTQ-PN ( $r=0.223, p=0.026)$, as well as with CTQ total scores $(\mathrm{r}=0.247, \mathrm{p}=0.013)$. Emotional withdrawal was positively associated with CTQ-EN ( $\mathrm{r}=0.317, \mathrm{p}=0.001)$ and CTQ-PN $(\mathrm{r}=0.246, \mathrm{p}=0.014)$, as well as with CTQ total scores $(\mathrm{r}=0.297$, $\mathrm{p}=0.003)$. The CTQ-EN score was positively correlated with the SANS total score $(\mathrm{r}=0.213, \mathrm{p}=0.033)$ and PANSS total score $(r=0.217, p=0.030)$. The analysis of dissociative symptoms in schizophrenia demonstrated that DES scores were positively correlated with hallucinatory behaviour $(\mathrm{r}=0.212$, $\mathrm{p}=0.034)$ and delusions $(\mathrm{r}=0.243, \mathrm{p}=0.015)$. CTQ-EN was significantly correlated with delusions $(r=0.208, \mathrm{p}=0.038)$. Additionally, SAPS total scores were significantly correlated with DES scores $(r=0.254$, $\mathrm{p}=0.011)$.

\section{Findings on the backward stepwise regression model}

The backward stepwise regression model is shown in table 4 . To select candidate variables to be included in the final model, one of the stepwise selection methods - the backward elimination method - was based on the likelihood ratio test statistics. The variables that had the least effect on the dependent variable $(p>0.05)$ were eliminated at each step, respectively, and the final model was reached. The set of independent variables that will be included in the model reached in step 4 was given in table 4 . In this study, the $\alpha$ significance level was determined as 0.05 . Considering that the input and output probability values are 0.05 and 0.10 in the backward stepwise elimination method, all variables (gender, DES, CTQEA, CTQ-EN, CTQ-PA, CTQ-PN, CTQ-SA and CTQ total scores) were included in the model in step 1 . The p-value of the CTQ-SA variable was determined to be the largest, and it was determined that it contributed the least to the model, and it was removed from the model and the second step was started. As a result of comparing the p-values again in the second step, the significance level of the CTQ-EA variable was found to be less than the other variables; and in this way, all variables were checked and another step was taken, and as a result of the fourth step, there were no variables to be removed from the model and the final model was created.

As seen in table 4, in the final model, the variables of gender, DES, CTQ-EN, CTQ-PA and CTQ-PN were determined as the variables with high contribution to the model, respectively. The final model obtained was created at the end of the fourth step. The results showed that gender, DES, CTQ-PA, CTQEN and CTQ-PN were significantly associated with schizophrenia presence. The odds ratio (OR) shows how many folds the effect of the determined scale variables on the model creates in patients with schizophrenia. It was observed that the variables with the highest OR were gender and CTQ-PA, respectively. Accordingly, when the CTQ-PA variable increases by 1 unit, its effect in evaluating patients with schizophrenia increases 1.729 times. When the CTQ-EN score variable, the third variable contributing the most to the model, increases by 1 unit, its effect in evaluating patients with schizophrenia increases 1.212 times. Male gender had a greater effect on the dependent variable than female gender. The logistic regression model established with the determined independent variables is as follows: patients $=-6.133+0.992 \times$ gender + $0.091 \times \mathrm{DES}+0.192 \times \mathrm{CTQ}-\mathrm{EN}+0.548 \times \mathrm{CTQ}-\mathrm{PA}-0.159 \times \mathrm{CTQ}-\mathrm{PN}$.

For the suitability of the model, the aim is to test whether all coefficients except the constant term are determinative on the model with the Hosmer-Lemeshow test. The hypotheses are as follows:

Ho: the parameters are decisive for the model.

$\mathrm{H}_{1}$ : the parameters are not decisive for the model.

The $\chi^{2}$ statistics for the fourth step were calculated as $\left(\chi^{2} \mathrm{~h}=6.157\right.$ and $\left.\mathrm{p}=0.630\right)$. Since $\chi^{2}$ calculation $<\chi^{2}$ table and $\mathrm{p}>0.05$, the Ho hypothesis was accepted and it was found that the variables included in the model were compatible for the model.

\section{Findings on the mediation model analysis}

The mediation model analysis was shown in figure 2. In this analysis, the presence and degree of influence of a variable (DES scores) mediating the possible effect of an independent variable (CT) on a dependent variable (presence of schizophrenia) were investigated. In figure 2, the direct effect of CT on schizophrenia was indicated by ' $c$ ', the effect of CT on DES by 'a'. The effect of DES on schizophrenia is indicated by ' $b$ '. The results 


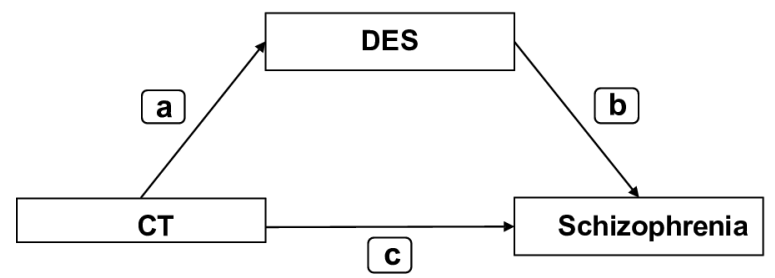

\begin{tabular}{|c|c|}
\hline $\begin{array}{c}\text { Regression } \\
\text { coefficient }\end{array}$ & P value \\
\hline $\mathrm{a}=0.4543$ & $<0.001$ \\
\hline $\mathrm{b}=0.8720$ & $<0.001$ \\
\hline $\mathrm{c}=0.8400$ & $<0.001$ \\
\hline
\end{tabular}

\section{Indirect effect of CT on schizophrenia $(a$ and $b)=0.0396$ \\ Direct effect of CT on schizophrenia (c) $=\mathbf{0 . 8 4 0 0}$ \\ Total effect of CT on schizophrenia $(a$ and $b+c)=0.8796$}

Figure 2 Path diagram of the mediation model analysis. CT, childhood trauma; DES, Dissociative Experiences Scale.

showed the DES-mediated effects of CT on the presence of schizophrenia with 'a and b'. The mediator variable effect ( $a$ and $b$ ) accounted for only a small portion of the total effect. The indirect effect of CT on schizophrenia (a and $b)=0.0396$.

\section{DISCUSSION}

\section{Main findings}

Our study determined that patients with schizophrenia had more history of CT than healthy controls. In addition, our results showed that childhood traumatic experiences were associated with dissociative, positive and negative symptoms in patients with schizophrenia. When the schizophrenia group was compared with the control group, a significant portion of those in the schizophrenia group were unemployed, had a low education level, were single, and had high rates of mental illness in their families. This is a predictable outcome consistent with the reduced functionality inherent in schizophrenia. ${ }^{20}{ }^{21} \mathrm{It}$ has been well established that individuals with schizophrenia smoke more frequently than the general population, and our findings also support this result. ${ }^{22} 23$

In the early stages, an individual's brain development can be impaired by genetic or environmental factors, which, in turn, create a predisposition for illness; in later life stages, schizophrenia symptoms can occur when this person is exposed to additional stressful environmental factors. It is thought that challenging experiences encountered in stress-sensitive developmental periods may affect the development of mental disorders due to the combined effects of genetic and other predisposing factors. In recent years, there has been increasing interest in whether exposure to CT increases the risk of schizophrenia. Some studies report that the history of CT is higher in patients with schizophrenia than in the general population. ${ }^{24}{ }^{25}$ Studies investigating the incidence rate of the history of CT in patients with schizophrenia have reported rates ranging from $45 \%$ to $85 \%{ }^{3}$ Our study, in agreement with the literature, found that CT was seen at a higher frequency in patients with schizophrenia compared to rates in healthy controls. In both the schizophrenia and control groups, the most common
CT was found to be EN (emotional neglect) and the least common one was found to be SA (sexual abuse). In the schizophrenia group, the total score and subscale scores of the CTQ scale, including CTQ-EA, CTQ-EN, CTQ-PA, CTQ-PN and CTQ-SA, were found to be higher compared with scores of the control group. The results of our study support the view that CT increases the risk of developing schizophrenia. Previous studies have similarly demonstrated that neglect and abuse specifically are observed more frequently during the childhood of patients with schizophrenia. $^{326}$

Varying results have been obtained in studies investigating the relationship between dissociative symptoms in patients with schizophrenia and positive and negative symptoms. One study reported a significant relationship between dissociative symptoms and positive symptoms, but found no correlation with negative symptoms. ${ }^{27}$ Another study stated that dissociative symptoms were only significantly correlated with hallucinatory behaviour and delusions, but not with other positive symptoms. ${ }^{28}$ In our study, a positive correlation was found between the total scores of positive symptoms and dissociative symptoms in patients with schizophrenia. Positive symptoms, hallucinatory behaviour and delusion scores from subscales and dissociative symptoms showed significant correlations. No significant relationship was found between negative symptoms and dissociative symptoms. This suggests that positive symptoms may be prominent in patients with schizophrenia who have dissociative symptoms or that dissociative symptoms may be observed more frequently in schizophrenia patients who have pronounced positive symptoms.

Dissociative symptoms may accompany schizophrenia and many psychiatric disorders. Studies conducted to date have reported a high rate of dissociative symptoms in patients with schizophrenia. In studies measuring the severity of dissociative symptoms in patients with schizophrenia, the mean DES score ranges from 10 to $30 .{ }^{13} 29$ In our study, the mean DES scores of patients with schizophrenia were found to be 21.47. In addition, the DES scores of the patients with schizophrenia were significantly higher than those of the healthy controls. We also 
observed significantly higher DES scores in patients with schizophrenia, which is similar to the literature findings.

Studies investigating the relationship between schizophrenia symptoms and CTQ have stated that positive symptoms are more common in individuals with a history of CTQ-SA and CTQ-PN than those who do not. ${ }^{30}$ In our study, a strong positive relationship was found between delusions from positive symptoms and CTQ-EN. Another study showed a relationship between CTQ and negative symptoms in patients with schizophrenia. In the same study, attention was drawn to the relationship between negative symptoms and CTQ-PN. ${ }^{31}$ Similar to the study results mentioned above, our study found a positive correlation with CTQ and negative symptoms such as passive/apathetic social withdrawal and emotional withdrawal. In addition, a positive correlation was found between negative symptoms' total scores and CTQ-EN. Negative symptoms, passive/apathetic social withdrawal and emotional withdrawal have been found to be associated with CTQ-EN and CTQ-PN. In addition, our study concluded that CTQ-EN was the trauma most closely associated with PANSS total scores, namely the severity of the disease.

Logistic regression analysis was performed with the scale scores and gender variable applied to the schizophrenia and control groups. Thereby, the creation of an explanatory model was attempted by defining the scales that are relevant in determining the risk of schizophrenia. In this binary logistic regression analysis, the schizophrenia group and control group were the dependent variables; the model was created by selecting gender, CTQ and its subscales, and DES as independent variables. Variables that were important in determining whether the risk of schizophrenia exists were male gender, DES, CTQ-EN, CTQ-PA and CTQ-PN. The OR shows how many folds the effect of the determined scale variables on the model creates in patients with schizophrenia. It was observed that the variables with the highest OR were gender and CTQ-PA, respectively. Accordingly, when the CTQ-PA variable increased by one unit, its effect in evaluating patients with schizophrenia increased 1.729 times. When the CTQ-EN score, the third highest variable contributing to the model, increased by one unit, its effect in evaluating patients with schizophrenia increased 1.212 times. Additionally, the effect of the male gender on the dependent variable was greater than that of the female gender.

Our study investigated the effects of CT on the presence of schizophrenia - either directly or via DES - with a model in which the independent variable was CT. Accordingly, it was understood that the mediator variable effect constituted a very small part of the total effect. In other words, the DES-mediated effect of CT on the presence of schizophrenia is lower than its direct effect. Dissociative symptoms may resemble psychotic symptoms in many cases, and even severe forms may be misdiagnosed as schizophrenia. However, evaluation for premorbid trauma is not routine in the psychiatric diagnostic process. Thus, including an assessment of traumatic experiences during the diagnostic stage may further the identification of alternative diagnoses or comorbid conditions and even affect the treatment process and form. In addition, considering the relationships separately between CT subtypes and DES may help better understand these interactions.

Results showing that CT may increase the risk of schizophrenia and its relationship with dissociative symptoms are remarkable. This may promote a bio-psycho-social model for the etiology of schizophrenia. While further detailed explorations of the relationship between CT and dissociation in individuals with schizophrenia are necessary, our findings of potentially different mediating functions show the points of intervention for individuals with CT. In the case of dissociative experience, it is more helpful to focus on schizophrenia and CT. According to CT, focusing on schizophrenia or dissociative symptoms could help for earlier diagnosis and reduce hospitalization. Therefore, training of mental health professionals in asking questions about CT may meet the need to provide appropriate psychosocial treatments to patients with schizophrenia who were abused or neglected as children.

\section{Limitations}

While the present study findings indicate strong relationships between the measures related to childhood trauma and schizophrenia, these results should be filtered with recognition of the study's limitations. First, this research was restricted by using retrospective self-reports, a limited sample size and the relatively questionable reliability of a single scale to evaluate CT. Second, most of the subjects in the schizophrenia group were patients in remission with a long-term psychiatric history who were being treated with antipsychotics. Results could have been different in firstepisode schizophrenia patients who had never received treatment. These characteristics prevent the generalisation of the findings put forth. However, we believe clinicians must be aware of the possible relationships between schizophrenia and CT and the high prevalence of dissociative symptoms in this at-risk group. Finally, we can make no definite statements regarding causal associations because of the study's cross-sectional nature.

Despite these limitations, the results of the present study highlight the distinct mediating effects of schizophrenia and dissociation in the context of their relationships with childhood trauma.

The results of our study indicate that CT and dissociative symptoms are seen at high rates in patients with schizophrenia. In addition, the findings of the relationship between CT and dissociative, positive and negative symptoms are also noteworthy. Therefore, it may be important for clinicians to assess trauma history during the psychiatric evaluation of patients with schizophrenia. More research is needed to better understand the complex relationship between dissociative symptoms and CT history in patients with schizophrenia. 
Contributors Study design-TTU and CH. Data collection and analyses-TTU and MB. Drafting the manuscript-TTU, MB and CH. Critical revision- $\mathrm{CH}$. Approval of the manuscript-all authors. Guarantor- $\mathrm{CH}$.

Funding The authors have not declared a specific grant for this research from any funding agency in the public, commercial or not-for-profit sectors.

Competing interests None declared.

Patient consent for publication Not required.

Ethics approval All subjects participating in the study were informed about the study, and their written consent was obtained. Family members and relatives of the patients, with whom we could interview, were also informed about the study. Before conducting the study, the approval of the ethics committee for the study was obtained from the Recep Tayyip Erdogan University Medical School Ethics Committee on 24 June 2016 with the decision number 2016/27.

Provenance and peer review Not commissioned; externally peer reviewed.

Data availability statement Data are available upon reasonable request. Not applicable.

Open access This is an open access article distributed in accordance with the Creative Commons Attribution Non Commercial (CC BY-NC 4.0) license, which permits others to distribute, remix, adapt, build upon this work non-commercially, and license their derivative works on different terms, provided the original work is properly cited, appropriate credit is given, any changes made indicated, and the use is non-commercial. See: http://creativecommons.org/licenses/by-nc/4.0/.

\section{REFERENCES}

1 Tandon R, Gaebel W, Barch DM, et al. Definition and description of schizophrenia in the DSM-5. Schizophr Res 2013;150:3-10.

2 Ered A, Ellman LM. Specificity of childhood trauma type and attenuated positive symptoms in a non-clinical sample. J Clin Med 2019;8:1537.

3 Lau S, Kirchebner J, Kling S, et al. Childhood maltreatment, psychopathology, and offending behavior in patients with schizophrenia: a latent class analysis evidencing disparities in inpatient treatment outcome. Front Psychiatry 2021;12:612322.

4 Popovic D, Schmitt A, Kaurani L, et al. Childhood trauma in schizophrenia: current findings and research perspectives. Front Neurosci 2019;13:274.

5 Wang Z, Xue Z, Pu W, et al. Comparison of first-episode and chronic patients diagnosed with schizophrenia: symptoms and childhood trauma. Early Interv Psychiatry 2013;7:23-30.

6 Uçok A, Bikmaz S. The effects of childhood trauma in patients with first-episode schizophrenia. Acta Psychiatr Scand 2007;116:371-7.

7 Friedberg A, Malefakis D. Resilience, trauma, and coping. Psychodyn Psychiatry 2018;46:81-113.

8 Oral R, Ramirez M, Coohey C, et al. Adverse childhood experiences and trauma informed care: the future of health care. Pediatr Res 2016;79:227-33.

9 Read J, van Os J, Morrison AP, et al. Childhood trauma, psychosis and schizophrenia: a literature review with theoretical and clinical implications. Acta Psychiatr Scand 2005;112:330-50.
10 Rosenberg SD, Lu W, Mueser KT, et al. Correlates of adverse childhood events among adults with schizophrenia spectrum disorders. Psychiatr Serv 2007;58:245-53.

11 Bendall S, Jackson HJ, Hulbert CA, et al. Childhood trauma and psychotic disorders: a systematic, critical review of the evidence. Schizophr Bull 2008;34:568-79.

12 Vogel M, Spitzer C, Barnow S, et al. The role of trauma and PTSDrelated symptoms for dissociation and psychopathological distress in inpatients with schizophrenia. Psychopathology 2006;39:236-42.

13 Ross CA, Keyes B. Dissociation and schizophrenia. Journal of Trauma \& Dissociation 2004;5:69-83.

14 Bernstein DP, Ahluvalia T, Pogge D, et al. Validity of the childhood trauma questionnaire in an adolescent psychiatric population. J Am Acad Child Adolesc Psychiatry 1997;36:340-8.

15 Sar V, Ozturk E, Ikikardes E. Validity of the childhood trauma questionnaire in Turkish population. Turkiye Klinikleri J Med Sci 2012;32:1054-63.

16 Bernstein EM, Putnam FW. Development, reliability, and validity of a dissociation scale. J Nerv Ment Dis 1986;174:727-35.

17 Sar V, Kızıltan E, Kundakcı T. Reliability and validity of the Turkish Version of Dissociative Experiences Scale-II. In: National psychiatry Congress proceedings full text book. , 1997: 33, 55-64.

18 Kay SR, Fiszbein A, Opler LA. The positive and negative syndrome scale (PANSS) for schizophrenia. Schizophr Bull 1987;13:261-76.

19 Kostakoglu E, Batur S, Tiryaki A, et al. Reliability and validity of the Turkish version of the positive and negative syndrome scale (PANSS). Türk Psikoloji Dergisi 1999;14:23-34.

20 Mohr P, Rodriguez M, Bravermanová A, et al. Social and functional capacity of schizophrenia patients: a cross-sectional study. Int J Soc Psychiatry 2014;60:352-8.

21 Aukes MF, Laan W, Termorshuizen F, et al. Familial clustering of schizophrenia, bipolar disorder, and major depressive disorder. Genet Med 2012;14:338-41.

22 de Leon J, Diaz FJ. A meta-analysis of worldwide studies demonstrates an association between schizophrenia and tobacco smoking behaviors. Schizophr Res 2005;76:135-57.

23 Li Y, Hou C-L, Ma X-R, et al. Nicotine dependence in communitydwelling Chinese patients with schizophrenia. Gen Psychiatr 2019;32:e100014.

24 Kosteletos I, Kollias K, Stefanis N. Childhood adverse traumatic experiences and schizophrenia. Psychiatriki 2020;31:23-35.

25 Loewy RL, Corey S, Amirfathi F, et al. Childhood trauma and clinical high risk for psychosis. Schizophr Res 2019;205:10-14.

26 Janssen I, Krabbendam L, Bak M, et al. Childhood abuse as a risk factor for psychotic experiences. Acta Psychiatr Scand 2004;109:38-45.

27 Ghoreishi A, Shajari Z. Reviewing the dissociative symptoms in patients with Schizophreniaand their association with positive and negative symptoms. Iran J Psychiatry Behav Sci 2014;8:13-18.

28 Spitzer C, Haug HJ, Freyberger HJ. Dissociative symptoms in schizophrenic patients with positive and negative symptoms. Psychopathology 1997;30:67-75.

29 Bob P, Mashour GA. Schizophrenia, dissociation, and consciousness. Conscious Cogn 2011;20:1042-9.

30 Ross CA, Anderson G, Clark P. Childhood abuse and the positive symptoms of schizophrenia. Hosp Community Psychiatry 1994:45:489-91.

31 Bennouna-Greene M, Bennouna-Greene V, Berna F, et al. History of abuse and neglect in patients with schizophrenia who have a history of violence. Child Abuse Negl 2011;35:329-32.

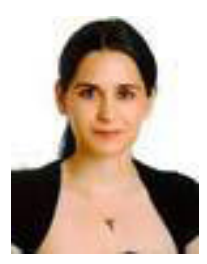

Tugce Taskin Uyan obtained a medical degree from Ondokuz Mayis University Faculty of Medicine in Turkey in 2009. Then, she received postgraduate training in psychiatry at the Medical School of Recep Tayyip Erdogan University in Rize, Turkey from 2014 to 2018. She is currently working as a psychiatrist at State Hospital in Rize, Turkey. Her main research interests include mood disorders and schizophrenia. 